\title{
Understanding intensities of angle-resolved photoemission with circularly polarized radiation from a $\mathrm{Cu}(111)$ surface state
}

\author{
M. Mulazzi* and G. Rossi \\ Dipartimento di Fisica, Università di Modena e Reggio Emilia, Via A. Campi 213/A, I-41100 Modena, Italy \\ and Laboratorio TASC-INFM-CNR, Area Science Park, S. S. 14, Km 163.5, I-34012 Trieste, Italy \\ J. Braun, J. Minár, and H. Ebert \\ Department Chemie und Biochemie, Universität München, Butenandtstrasse 5-13, D-81377 München, Germany \\ G. Panaccione, I. Vobornik, and J. Fujii \\ Laboratorio TASC-INFM-CNR, Area Science Park, S. S. 14, Km 163.5, I-34012 Trieste, Italy \\ (Received 19 August 2008; revised manuscript received 27 January 2009; published 15 April 2009)
}

\begin{abstract}
The $\mathrm{Cu}(111)$ Shockley surface state has been widely measured by photoemission spectroscopy as a prototypical spectroscopy experiment to show parabolic dispersion and energy resolution. Intensity asymmetries have been observed but never interpreted for lack of systematic evidence and theoretical background. Here we report an extended study of the angle-resolved photoemission intensity of the $\mathrm{Cu}(111)$ Shockley surface state as measured in different experimental geometries, variable synchrotron light polarization, and at different photon energies in the $18-70 \mathrm{eV}$ range. Strong asymmetries appear with respect to the $\Gamma$ point. We provide an interpretation of the observed intensity asymmetry based on the symmetry of the face-centered-cubic lattice and by means of density-functional theory calculations: we conclude that the origin of the asymmetry is connected with the contribution of the $p$-to- $d$ photoemission channel to the total photoemission intensity, opening an unexpected view on final-state matrix element effects.
\end{abstract}

DOI: 10.1103/PhysRevB.79.165421

PACS number(s): 73.20.-r, 79.60.-i

\section{INTRODUCTION}

Photoemission intensities in angle-resolved experiments reflect the radial features of the initial-state wave functions via the photoemission matrix elements. Angle-resolved photoemission spectroscopy (ARPES) experiments can address therefore a more complete description of the electronic structure, beyond $E$ vs $k$, energy distribution curves, momentum distribution curves, and constant energy maps, provided that the effects of the experimental geometry are fully understood. Symmetry rules are in fact at work for selecting finalstate intensities when measuring in proximity of mirror planes while no simple on/off rules are at work in the general case.

Recent photoemission experiments on ferromagnetic surfaces showed how complex it is to disentangle the magnetic dichroism effects in ARPES from the matrix element effects that are always present, independently of the symmetry reduction determined by magnetization. ${ }^{1,2}$

We report here on systematic measurements of the Shockley surface state of $\mathrm{Cu}(111)$ that has attracted considerable theoretical and experimental effort since it represents a case study for many important aspects of solid-state physics such as spin-orbit coupling (SOC), electron-electron, and electron-phonon interactions. ${ }^{3-6}$

The $\mathrm{Cu}(111)$ Shockley surface state has been abundantly measured because it lies in the $L$ bulk-band gap and is mainly formed by $s p$-type orbitals featuring nearly-freeelectron dispersion law. Therefore it has been "well understood" in terms of $E(\vec{k})$ as a textbook example of the freeelectron final-state approximation for excitation at UV-soft $\mathrm{x}$-ray energies. Interestingly the intensity in the photoelec- tron distribution is asymmetric with respect to the $\Gamma$ point but this fact, despite appearing in previously published data, has always been neglected in the analysis. . $^{7-9}$

The rationale of the present study is therefore the analysis of the photoemission intensity distributions of a "simple" initial state (weak hybridization, parabolic dispersion) as a function of photon energy in the range typical of surfacestate spectroscopy, i.e., $18-70 \mathrm{eV}$, and with all polarization states as a component of relevant experimental geometries.

Early photoemission spectroscopy (PES) experiments showed that the integrated photoemission intensity from the surface states of noble metals depends strongly on the photon energy. These findings were interpreted ${ }^{10,11}$ in terms of the dependence of the photoemission cross section on the density of the final states: the higher the density of free states for the excited photoelectrons is, the more probable is the photoemission process and then the total measured intensity.

The use of circular polarization to excite photoemission from valence bands was applied for the first time in the 1980s (Refs. 12-14) to $\operatorname{Ag}(100)$ and $\operatorname{Pt}(111)$. In these works spin-polarized photoelectrons were detected from nonpolarized samples when circularly polarized photons are tuned in energy to excite the hybridized electron bands. In all these works the assumption of a parabolic final-state band was taken to support the experimental data analysis.

In this work we concentrate on the measurement and analysis of the $k$-space resolved photoelectron intensity distribution (PID) obtained using mainly circular polarization and several different photon energies.

With the experimental PID being unexpectedly rich of asymmetries and energy as well as polarization dependences, we also performed photoemission calculations that take into 


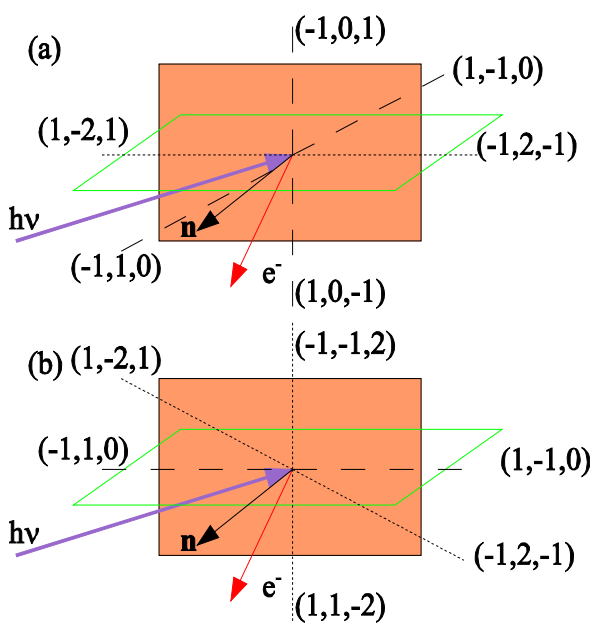

FIG. 1. (Color online) Sketch of the experimental geometries. The photon beam (violet or dark gray arrow), the electron momentum (red or light gray arrow), and the surface normal are kept in the same scattering plane (fine green line). The thinly and the coarsely dashed lines represent the crystal high-symmetry planes, respectively: the finely dashed ones represent mirror planes and coarsely dashed ones represent nonmirror planes of the $\mathrm{Cu}(111)$ surface. In (a) a mirror plane coincides with the scattering plane (photoemission measures bands along the $\Gamma-M$ direction) so that the dipole selection rules for the matrix elements are valid. This is not the case for (b) since no mirror plane is parallel to the scattering plane (ARPES measures taken along the $\Gamma-K$ direction). Geometry (a) is changed to (b) by rotating the crystal about the surface normal by $30^{\circ}$.

explicit account the symmetry of the final-state wave functions in the evaluation of the matrix element.

\section{EXPERIMENTAL DETAILS}

Experiments have been performed at the low energy branch of the APE-INFM beamline at the Elettra storage ring in Trieste, providing linearly ( $p$ and $s$ ) as well as circularly (left-handed and right-handed) polarized photons in the 7-130 eV energy range by means of an Apple II quasiperiodical undulator. The design of the APE beamline is such that the beam footprint on the sample is the same for all energies and polarizations delivered from the undulator source. We estimate that the circular polarization rate is around $65 \%$ for both helicities (within 1\%) and only weakly varying as a function of the photon energy. The Scienta SES 2002 electron analyzer allows a parallel detection of the photoelectron in a $14^{\circ}$ angular window with an angular resolution better than $0.2^{\circ}$.

Figure 1 depicts the geometry of the experiment: the data presented at normal emission have been obtained with light impinging at an angle of $43^{\circ}$ from the analyzer axis while for the data taken at about normal incidence the emission angle was about $43^{\circ}$; we used 12 energies between $h \nu=18 \mathrm{eV}$ and $h \nu=70 \mathrm{eV}$, and circular left $\left(C^{-}\right)$and right $\left(C^{+}\right)$polarizations always keeping the plane containing the $\Gamma-M$ (or $\Gamma-K$ ) direction coincident with the scattering plane [condition obtained by orienting the sample by means of the low-energy electron diffraction (LEED) pattern as measured in situ].

All measurements were done at $300 \mathrm{~K}$, and the overall (temperature dominated) energy broadening was $100 \mathrm{meV}$. As a consequence, the surface-state peak width (at the $\Gamma$ point) is $\approx 90 \mathrm{meV}$, which is in agreement with the data of Baumberger et al. ${ }^{15}$ Repeated cycles of $\mathrm{Ar}^{+}$sputtering at 1 $\mathrm{keV}$ and annealing at $500{ }^{\circ} \mathrm{C}$ led to clean surfaces whose crystalline long-range order was checked by LEED. No evidence of contamination was found immediately after the cleaning procedure while carbon and oxygen peaks were clearly visible $36 \mathrm{~h}$ later and correspondingly the $\mathrm{Cu}$ surfacestate intensity was reduced by $\sim 30 \%$.

Dealing with intensities and photon energy dependence, a proper normalization of the PES maps was needed: in order to remove any extrinsic contribution of the detector to the photoemission intensities, we measured the complete valence-band spectra, including the secondary background at low kinetic energies, in the whole angular range of the surface state. We then divided the surface-state maps by a momentum distribution curve, obtained by averaging over 0.5 $\mathrm{eV}$ the intensity of the secondary background as far as possible from both the $d$ and $s$ - $p$ bands.

\section{THEORETICAL DETAILS}

It is widely accepted to interpret a measured photoemission spectrum in noncorrelated materials by referring to the results of band-structure calculations that are based on density-functional theory (DFT) in the local-density approximation (LDA). ${ }^{16,17}$ In order to achieve a reliable interpretation of the experimental spectra, the wave vector and energy dependence of the photoemission matrix elements has to be accounted for. This implies that the selection rules, multiplescattering processes, ${ }^{18}$ and an accurate description of the surface barrier have to be included in the ground-state, finalstate, and matrix element calculations. These are carried out in the so-called one-step model of photoemission as originally proposed by Pendry and co-workers ${ }^{18-20}$ whose main idea of the one-step model is to describe the excitation process, the transport of the photoelectron to the crystal surface, as well as the escape into the vacuum as a single-quantummechanically coherent process. ${ }^{21}$ In order to calculate relativistic photoemission intensities, we start in solving the Dirac equation that can be obtained from the relativistic generalization of density-functional theory. ${ }^{22,23}$ It can be solved using a phase-functional ansatz ${ }^{24}$ generalized to the relativistic case. ${ }^{25-28}$ From this solution it is easy to define the atomic scattering matrix $\Gamma$ for a single-ion-core potential together with the wave functions for the initial and for the final state. The atomic scattering matrix $\Gamma$ together with the crystal geometry determines the scattering matrix for a single layer. By means of layer-doubling techniques the so-called bulk-reflection matrix can be calculated, which gives the scattering properties of a semi-infinite stack of layers. Finally applying the spin polarized low electron energy diffraction theory ${ }^{29-37}$ we are able to derive the final state and the initial state for the semi-infinite crystal. Four different terms build up the relativistic photocurrent, the atomic, the intralayer, the interlayer, and the surface contributions, whose detailed ex- 
pressions and derivations can be found elsewhere. ${ }^{19,20,38,39}$

Lifetime effects in the final and initial states have been included in our analysis in a phenomenological way using a parametrized complex inner potential $V_{o}(E)=V_{o r}(E)$ $+i V_{o i}(E)$. Herein the real part serves as reference energy inside the crystal with respect to the vacuum level. For the final and initial states, constant imaginary parts $V_{o i}\left(E_{2}\right)$ $=2.5 \mathrm{eV}$ and $V_{o i}\left(E_{1}\right)=0.015 \mathrm{eV}$ have been chosen. The bulk potential which we used in the photoemission calculations results from a fully relativistic spin polarized relativistic Korringa-Kohn-Rostoker calculation. ${ }^{40}$ A realistic description of the surface potential is given through a RundgrenMalmström barrier ${ }^{41}$ while the value of the work function $\phi=4.88 \mathrm{eV}$ is taken from previous works. ${ }^{39}$ In such work a method describing a physically significant choice of the barrier potential can be found. The parameters describing the potential barrier used for a best fit to the data are $z_{I}=$ -2.047 a.u., $z_{A}=-3.611$ a.u., and $z_{E}=-0.522$ a.u..

\section{RESULTS}

\section{A. Spectroscopic measurements}

The average (over photon energy and polarization) parameters characterizing the $\mathrm{Cu}(111)$ surface state extracted from our spectra are $E(\Gamma)=0.35 \pm 0.02 \mathrm{eV}$ as the value of the binding energy at the $\Gamma$ point, $k_{F}=0.193 \pm 0.013 \AA^{-1}$ for the Fermi vector, and $R=0.4 \pm 0.02$ for the electron effectivemass ratio. These parameters were already known from similar experiments carried out at $30 \mathrm{~K}$ using a Helium lamp as photon source. ${ }^{42} \mathrm{We}$ assign the discrepancy between the literature data and those reported here to the different sample temperature, as suggested by McDougall et al. ${ }^{43}$

The $\mathrm{Cu}(111)$ surface state has negligible hybridization with other electron bands. The presence of a strongly asymmetric photoelectron intensity distribution is not expected based on our current understanding of photoemission from solid surfaces. The free-electron final-state approximation can be "safely" used to determine the dispersion law, resulting in a parabola with accepted parameters. But the same approximation does not explain the PID. A better analysis of the transitions between initial and final states is needed to interpret the origin of the PID asymmetries.

The four possible causes of the nonzero value of the intensity in the "forbidden" $s$-polarized experimental ARPES data of Fig. 2 are (i) the presence of $d$ bands in the initial state, (ii) quadrupolar transitions, (iii) indirect transitions, and (iv) incomplete $s$ polarization of the incident light. The contribution of the $d$ orbitals to the initial state is indeed negligible [less than 1\% (Ref. 44)], and quadrupolar transition are very weak at low energy (less than $0.1 \%$ ). Nonzero intensity for $s$ polarization can be due to electrons thermally excited (indirect transitions) from $k$-space locations away from the mirror plane. Such electrons, not subject to the dipolar selection rules, may give a finite contribution to the photoemission signal. Since we only measured at room temperature, we cannot quantify indirect-transition intensities. We can anyhow ascribe the $6 \%$ intensity to a residual $p$ polarization in the nominally $s$ radiation.
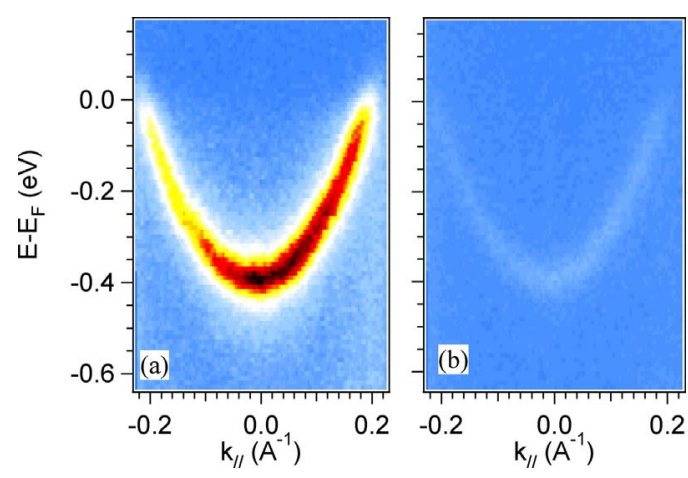

FIG. 2. (Color online) Angle-resolved photoemission maps of the $\mathrm{Cu}(111)$ surface state measured with $h \nu=18 \mathrm{eV}$, and (a) $p$ and (b) $s$ polarizations plotted in a color scale ranging from blue (lowest intensity), white, yellow, red, and black (highest intensity). The intensity ratio between the $s$-to- $p$ polarization data is a function of momentum (or binding energy) but it assumes a maximum value of $6 \%$ that can be addressed to incomplete $s$-polarized radiation.

This is readily observed in Fig. 2, an angle-resolved photoemission map measured at $18 \mathrm{eV}$ with $p$ - and $s$-polarized lights. Strong intensity is only measured with $p$ polarization while the $s$-polarization spectrum has a maximum intensity equal to only about $6 \%$ of the $p$ spectrum.

Figure 3 shows a selection of ARPES maps at several photon energies and for the two circular polarizations. Clear differences between the positive and negative momentum sides of the images are observed as well as the differences at some photon energies between the data taken with the two circular polarizations. While at the two lowest energies the spectral weight lies mostly at positive $k$ values for both polarizations, at $h \nu=44 \mathrm{eV}$ the PID shows largest intensity at negative $k$ values with respect to the $\Gamma$ point. The main effect is connected with the change in excitation energy but also the polarization plays a role in shifting the intensity maximum, enhancing or reducing the asymmetry of the PID.

As already mentioned we also measured ARPES maps of the surface state oriented with the plane containing the $\Gamma-K$ direction, coincident with the scattering plane. In this configuration, the mirror plane containing the $\Gamma-M$ direction is perpendicular to the scattering plane. As a consequence, no difference in the spectra between left and right polarizations is allowed unless an experimental misalignment is present. As seen in the ARPES maps presented in Fig. 4 in this configuration, no transfer of spectral weight caused by the change in polarization is observed while a consistent transfer is visible from the corresponding data taken in the $\Gamma-M$ plane presented in Fig. 3.

The data show that the PID evolves with energy and polarization in an unpredictable way, not expected in the freeelectron final-state approximation. The effect of the polarization is to enhance the intensity shift toward positive or negative $k$ values.

In order to evaluate the possible influence of geometrical factors on the left-right intensity unbalance, we also examined the ARPES intensity of the surface state at normal incidence in the second Brillouin zone and the results of these measurements are shown in Fig. 5. 


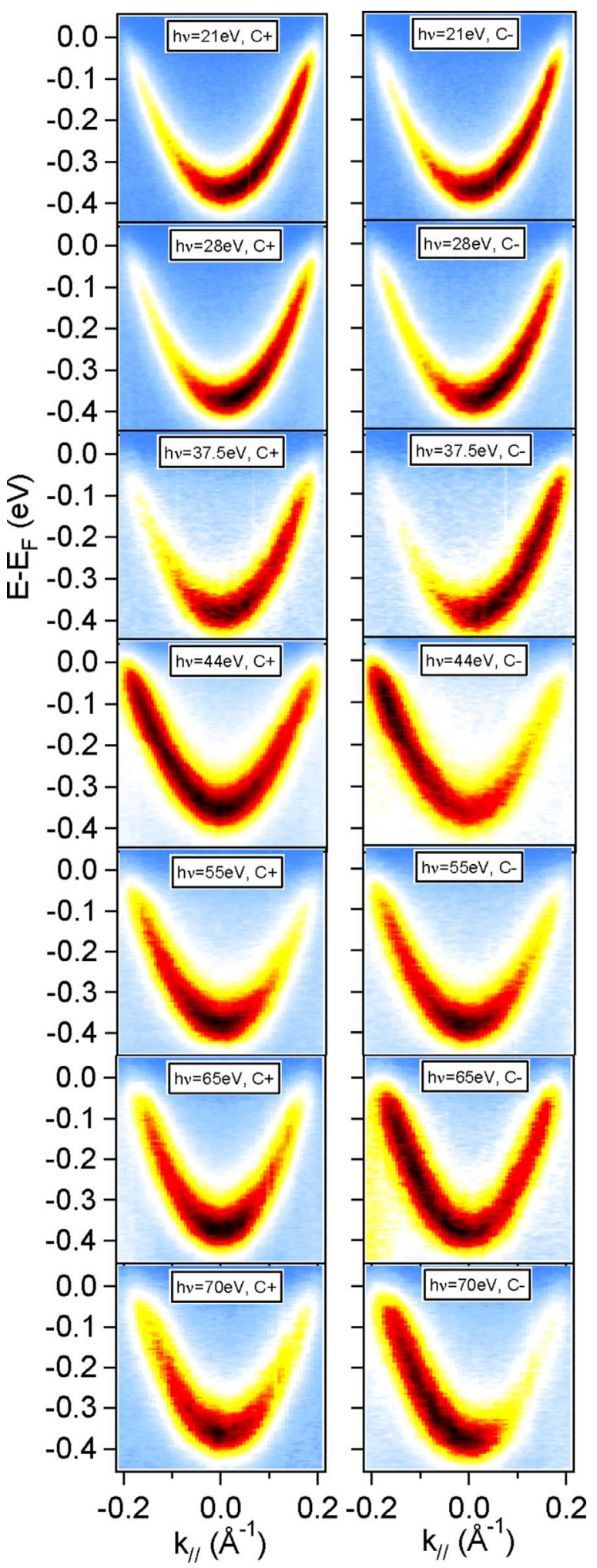

FIG. 3. (Color online) Selection of experimental angle-resolved photoemission maps of the $\mathrm{Cu}(111)$ Shockley surface state at different photon energies and polarizations. As noticed the PID follow a complex behavior due to matrix element effects. This kind of intensity distribution is not expected on the basis of a free-electron final-state approximation.
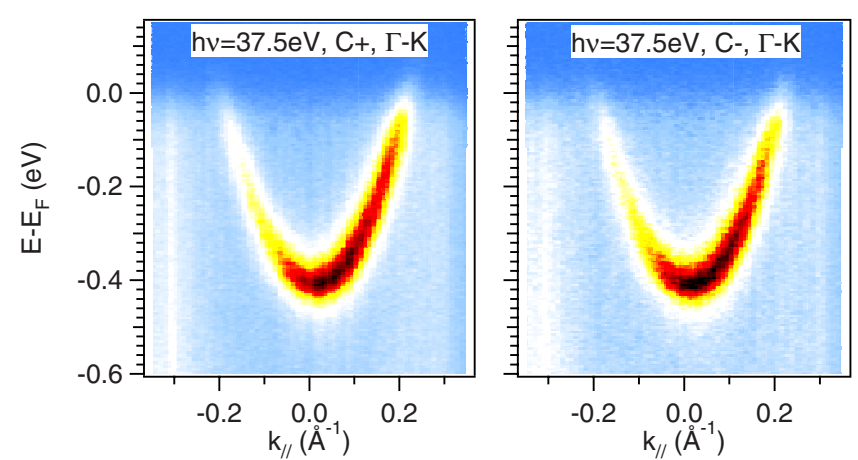

FIG. 4. (Color online) The ARPES map taken with the $\Gamma-K$ direction coincident with the scattering plane for the two circular polarizations. No spectral weight transfer is observed as expected since the mirror plane containing $\Gamma-M$ is perpendicular to the scattering plane forbidding a left-to-right asymmetry in the PID.

Also the data in the second Brillouin zone are affected by a strong asymmetry that does not depend on the emission angle and is weakly depending on the photon energy. This proves that the experimental geometry cannot be the only cause of the PID asymmetry on the surface state but it certainly contributes to it.

\section{B. One-step calculations}

In Fig. 6 we present calculated ARPES maps of the $\mathrm{Cu}(111)$ Shockley state excited with photon energies as the experimental ones for both polarizations. The $E$ versus $\mathbf{k}_{\|}$ plot of the surface-state intensity is presented in the same color scale of the experimental data (as detailed in the caption of Fig. 2). The main surface-state parameters inferred from the calculations are $E(\Gamma)=0.43 \mathrm{eV}$ as the value of the binding energy at the $\Gamma$ point, $k_{F}=0.205 \AA^{-1}$ for the Fermi vector, and $R=0.4$ as the effective-mass ratio. These values, considering the above-mentioned temperature dependence of the binding energies, are in very good agreement with the experimental values.
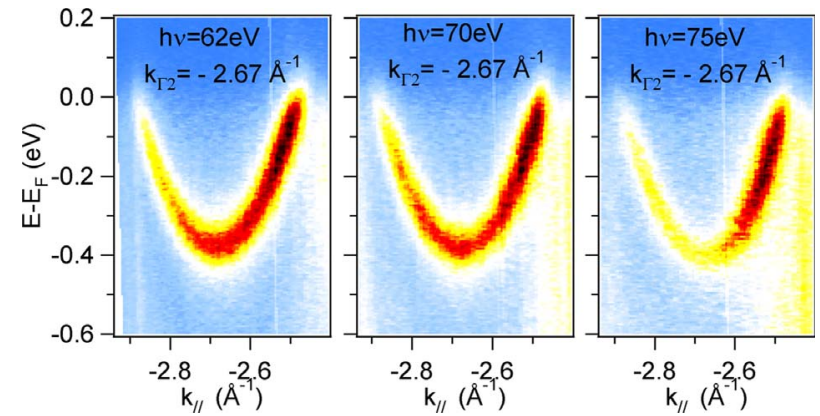

FIG. 5. (Color online) ARPES maps of the $\mathrm{Cu}(111)$ surface state measured with linear $p$ polarization at three photon energies $(h \nu$ $=62,70$, and $75 \mathrm{eV}$ ) and three angles: $95^{\circ}$ (left panel), $90^{\circ}$ (central panel), and $88^{\circ}$ (right panel), yielding always a nonsymmetric spectral weight, with more intensity on the right side of the mirror plane (positive value of $\mathbf{k}_{\|}$). The intensity at the sides of the surface state comes from the bulk-derived $s p$ bands that approach by means of $k$-perpendicular dispersion of the surface states at high photon energy. 


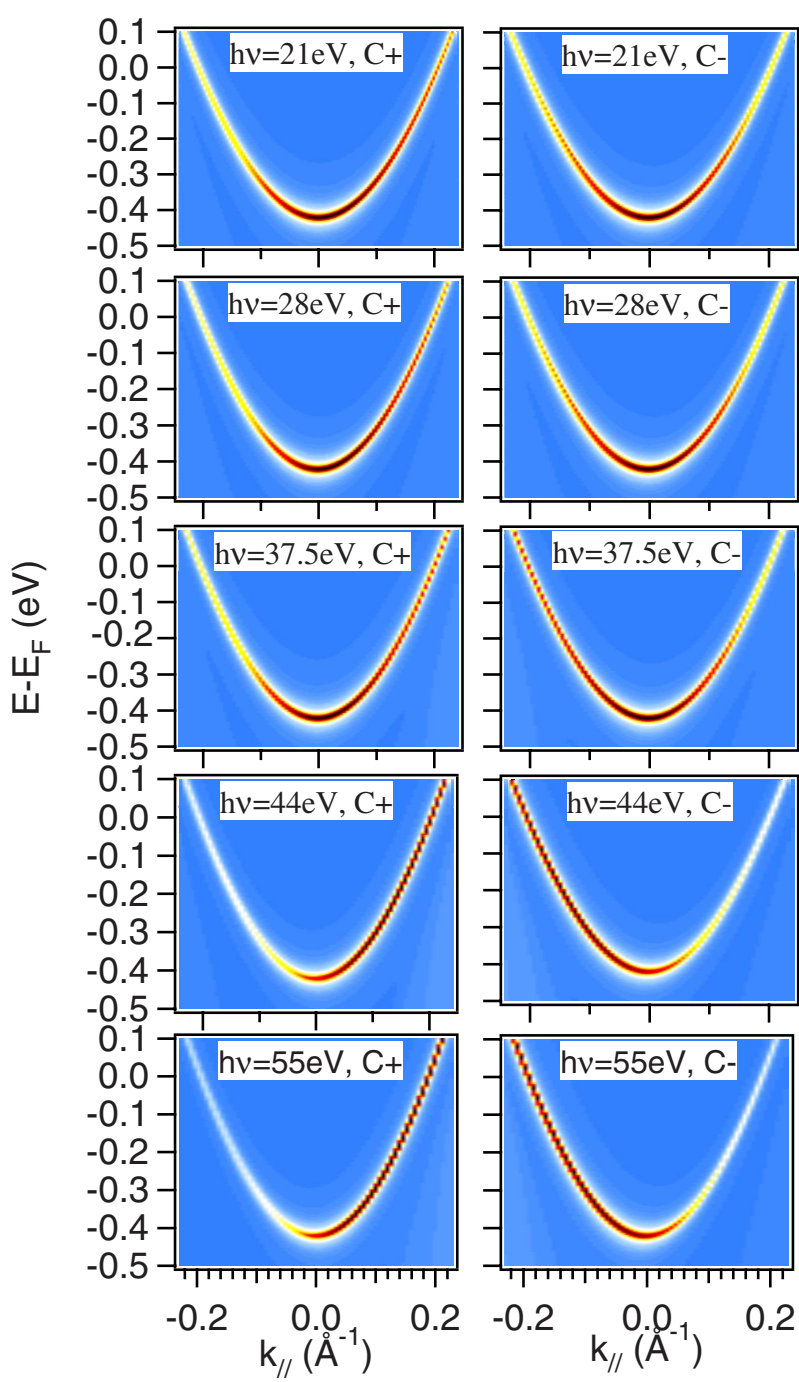

FIG. 6. (Color online) Angle-resolved photoemission maps of the $\mathrm{Cu}(111)$ surface state calculated at the same photon energies as the experimental data of Fig. 3 for right circular polarized $\left(C^{+}\right.$, left side) and left circular polarized lights $\left(C^{-}\right.$, right side). Strong variation in the PID are observed in the calculated spectra albeit the correspondence to the experiment is only qualitative as explained in the text

From the inspection of Fig. 6, it is possible to see that the ARPES maps calculated at energies below $h \nu=44 \mathrm{eV}$ for $C^{+}$ polarization have the PID centered on the right side of the image (positive wave vectors). This fact is also observed in the corresponding experimental data. The calculations done with $C^{-}$polarization show almost no asymmetry and a slight trend shifting the spectral weight to the left side of the image (negative wave vectors), which is at variance with the experiment.

However, the situation is reversed at higher energies, where the calculations done with $C^{-}$agree better with the data than those done with $C^{+}$. In fact at $h \nu=44 \mathrm{eV}$ and $h \nu$ $=55 \mathrm{eV}$ the calculations done with $C^{-}$lie on the same side of the image as the experimental data while the $C^{+}$polarization data lie on the opposite side.

The calculations present the PID asymmetry and the enhancement of the polarization effects at photon energies above $40 \mathrm{eV}$, both features also present in the experimental data. In fact, the experimental behavior is even richer than the theoretical one, with the PID maxima shifting from left to right more than predicted by theory.

From the theoretical point of view, we interpret the effect of changing the phase of the polarization on the photoemission maps as a shift in the position of the "center of gravity" of the PID while the change in photon energy increases the distance between the two centers of gravity. In the calculated spectra we see that the evolution of the PID with the polarization change is weak at low photon energies while it becomes stronger above $\approx 40 \mathrm{eV}$, i.e., the centers of gravity of the ARPES maps are close to each other below $40 \mathrm{eV}$, then further separate from each other at higher photon energy.

Such a trend is even more explicit in the calculated PIDs at energies higher than $h \nu=50 \mathrm{eV}$, shown in Fig. 7, leading us to conjecture (justified by the general behavior of the photoemission cross sections as functions of the energy) about the different transition probabilities which are connected with the corresponding final-state bands available when increasing the excitation energy.

We remark the utter importance of the correct description of the final states for the discussion of matrix elements. It is known that the LDA better reproduces the experimental data at smaller excitation energies, implying a good description of the final states near the Fermi level. Thus, the calculations fail to reproduce the high excitation energy PID asymmetry while feeling at the same time the sensitivity to the different transition probabilities.

\section{DISCUSSION}

Up to the observations, and with the incertitude, listed in the description of Fig. 2, all is consistent with the standard understanding of matrix element effects in photoemission experiments with polarized light and a geometry involving mirror planes which predict no PID asymmetry. The possible effects of excitation in non-normal-incidence geometry can be excluded from being relevant in determining the observed PID from the comparison of first and second Brillouin-zone data of Fig. 5, which were measured at normal incidence, confirming the PID asymmetry.

General causes of PID can be inferred considering the symmetries of the fcc lattice cut along the (111) plane. We must expect intensity asymmetries in the photoemission spectrum for bulk bands since in the [110] mirror plane the atoms do not stack along the (111) direction (corresponding to the $\Gamma-M$ in the reciprocal space) but in an asymmetric way with respect to the latter. Thus in the [110] plane the positions of the atoms at the right of the (111) direction are not mirror reflected into the left side and vice versa. As a result of this structural detail, a left-to-right asymmetry in the initial-state electron density and in the PID with respect to the normal-emission direction ( $\Gamma$ point of the Brillouin zone) must appear when bulk bands are measured keeping the $\Gamma-M$ direction in the scattering plane, as drawn in Fig. 1. This effect is readily observed in Fig. 8 in which the experimental data taken at two energies and polarizations are represented. As for the surface-state data an energy dependent asymmetric PID is present. 

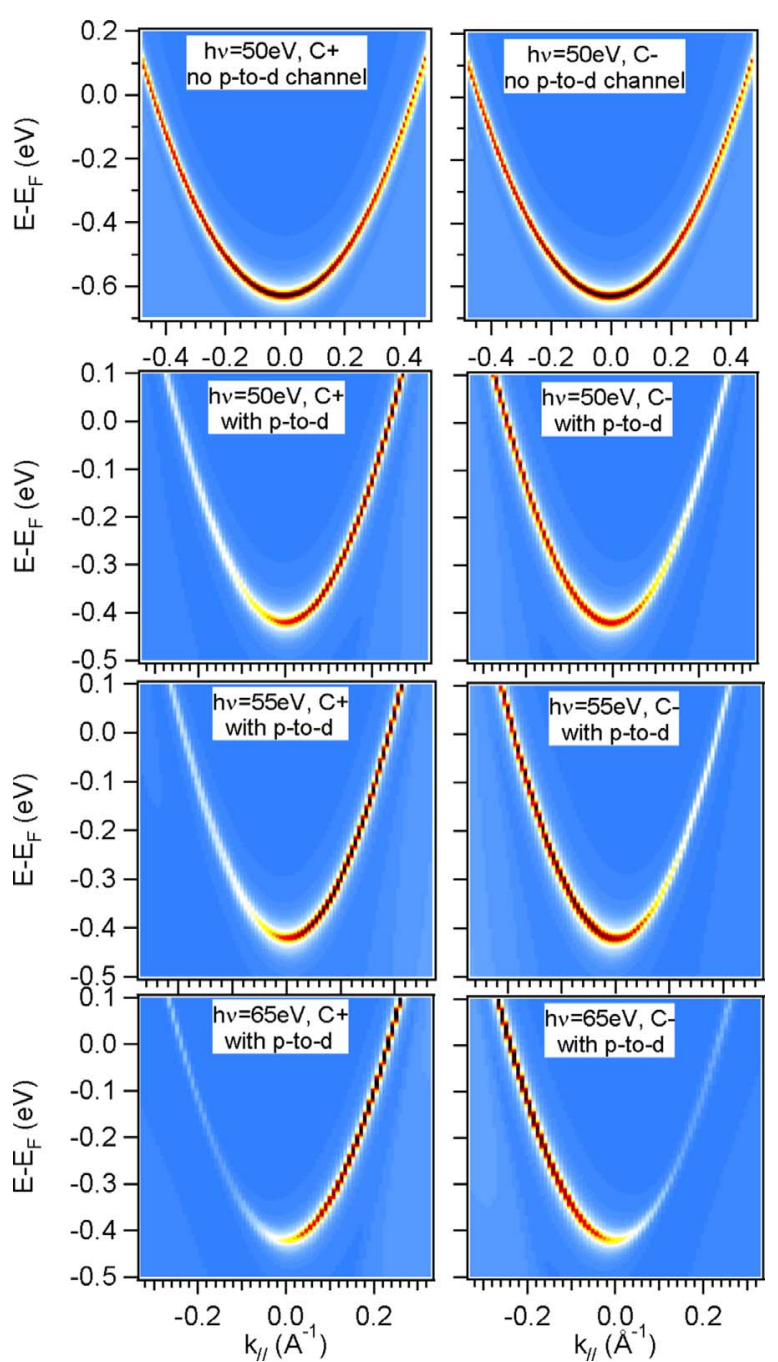

FIG. 7. (Color online) Angle-resolved photoemission maps of the $\mathrm{Cu}(111)$ surface state calculated at $h \nu=50$ (first and second row), 55 (third row), and $65 \mathrm{eV}$ (fourth row) for right circular polarized $\left(C^{+}\right.$, left side) and left circular polarized lights $\left(C^{-}\right.$, right side). In the first two panels from the top, the photoemission maps are calculated suppressing all the $d$ bands in the initial states (truncated basis set). This is also the reason for the difference in the binding energy and effective mass with respect to the calculations including all the possible electron bands (truncated basis set), as in the other six photoemission maps. In the calculations done with the full basis set the centers of gravity of the PID progressively separate from each other when the photon energy is increased while those with the truncated basis set has no asymmetry observed.

These symmetry considerations do not apply to surface states because they are localized in the very first atomic layers. In the limit of a surface state confined in only one layer, the argument above would not hold because of the lack of atomic stacking and then the electronic states would have a sixfold symmetry about the $\Gamma$ point. In the intermediate case of confinement the lattice symmetry may give some effect, i.e., there can be some left-to-right asymmetry of the PID induced by the lattice structure. This very general argument is consistent with the observed PID in the experimental spectra, both as measured with circular polarization at normal
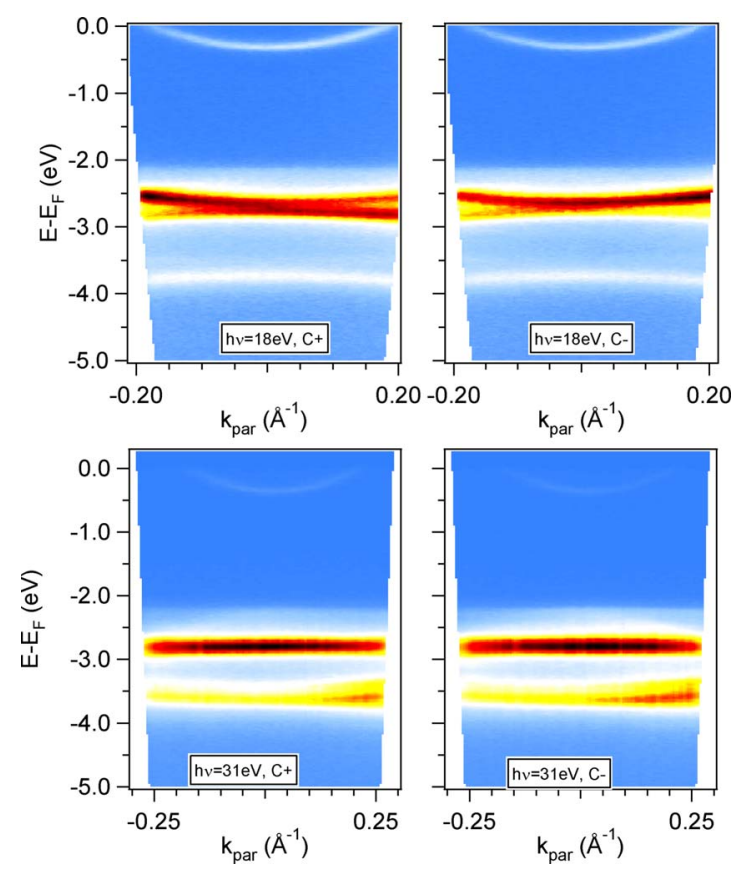

FIG. 8. (Color online) Valence-band data measured along the $\Gamma-M$ direction using two photon energies $(18 \mathrm{eV}$ in the top part and $31 \mathrm{eV}$ in the bottom part), and $C^{+}$(left part) and $C^{-}$(right part) circular polarizations. The PID is affected by strong matrix elements which are photon, energy, and angle dependent.

emission and with linear polarization at normal incidence. Nevertheless these considerations do not predict the magnitude or sign of the PID asymmetry, its energy dependence or its polarization dependence.

The trends of PID derived from the DFT calculations give further insight toward the understanding of the experiments when the types of wave functions constituting the surface state are considered. The photon energy and polarization dependence of the PID become higher with increasing photon energy, as seen in Fig. 7. Due to the $s p$-type character of the Shockley surface state, both $s$ - $p$ type and $p$ - $d$ type dipole allowed transitions contribute to the photoemission intensity. In order to disentangle the $s-p$ and the $p$ - $d$ contributions in the photoemission, we calculated a PID by suppressing the $p$ - $d$ channel: the result is also presented in Fig. 7 showing ARPES maps of the $\mathrm{Cu}(111)$ surface state as calculated at $h \nu=50 \mathrm{eV}$ for $C^{+}$and $C^{-}$polarizations with and without $p$-to- $d$ matrix elements. In contrast to the (complete) calculated spectra, no asymmetry in the PIDs is predicted when the $p$ - $d$ channel is neglected.

Furthermore, the intensity structure is shifted to higher binding energy and the dispersion is less pronounced as compared to the complete calculation. This is simply a consequence of the neglected $p$ - $d$ transitions. The relevant result of suppressing the intensity asymmetry gives strong indication that the $p$ - $d$ transition represents indeed the excitation channel responsible for the measured left-right polarization dependence of the surface-state photoemission intensity. This is the main insight obtained from the present theoretical analysis and we take it as the relevant element for understanding the experimentally observed behavior: it is the $p$ - $d$ 
channel that generates the large PID asymmetry reversal upon circular polarization helicity inversion. The calculated effects are consistent in the trend in energy but larger than the experimental observations. In fact, beyond the relevant trend, the agreement between calculations and experiment at each energy is poor: this can be attributed to a number of facts that we will discuss below. The calculated trend and the absence of effects when the $p$ - $d$ channel is switched off are very suggestive that the symmetry of final states available for the photoemission plays the main role in the PID at energies of $18-65 \mathrm{eV}$.

We stress that the hybridization between the ground surface state and the bulk bands is negligible, as seen in Refs. 42 and 44: the surface state lies at the $L$ bulk-band gap. One can understand as a consequence that the surface-state PID asymmetry is completely decoupled from the $d$ band photoemission asymmetry, as seen in Fig. 8. The origin of the observed PID can be understood in the coupling between the initial-state bands and the final-state bulk bands above the Fermi level: if the latter are of $p$ type only, then no asymmetric PID is found. The asymmetric PID is an on/off effect that appears whenever $d$-type final states are available for the photoemission process. The calculated effect is a priori independent on surface-bulk $s p$ states hybridization (expected negligible).

The differences between the experimental and theoretical PID, apparent when comparing data for the same energy and polarization, may be ascribed to: (a) poor description of the highly energetic final-state bands by the density-functional theory in the LDA. Such effects are well known and often have to be considered even for relatively low excitation energies. (b) Poor description of the exact hybridization of the Shockley surface state. (c) Further hybridization of the measured Shockley surface state due to defects (steps, kinks) or contaminants.

The PID appears in fact as an extremely sensitive measure of the actual hybridization state of the initial-state wave function, and may turn out to be a useful probe for studying surface chemistry or surface phase transitions.

\section{SUMMARY}

In our study on $\mathrm{Cu}(111)$, we have observed a complex behavior of the photoemission PID of the Shockley surface state as a function of light polarization and energy, which could not be expected on the basis of standard phenomenological descriptions of the photoemission process and the free-electron final-state approximation. One-step-model calculations including a realistic surface barrier with parameters given from the experiment are able to predict phenomena that are consistent with the experimental trends. The main phenomenon determining the photoemission intensity asymmetry of the Shockley surface state as a function of $\mathbf{k}_{\|}$has been identified as a strong matrix element effect connected to the $p$ - $d$ transition channel from the $p$-type orbital of the surface state into $d$-type final-state bands of $\mathrm{Cu}$. This surprisingly shows the failure of the free-electron final-state approximation when analyzing the photoemission intensities, this fact occurring even for final states at energies of several tens of electron volts above the Fermi level, i.e., in a spectroscopy regime where the dispersion law determination is widely accepted to be given by the plane-wave final states.

\section{ACKNOWLEDGMENTS}

The experiments were supported by CNR-INFM. For the theoretical work financial support from the Deutsche Forschungsgemeinschaft is gratefully acknowledged as well as the BMBF within the Verbundprojekt "Elektronische Struktur und Photoemission von hochkorrelierten intermetallischen seltenen Erdverbindungen" (Contract No. 05605MPA0).

\footnotetext{
*mulazzi@spring8.or.jp

${ }^{1}$ M. Mulazzi, Ph.D. Thesis, University of Modena e Reggio Emilia, 2006.

${ }^{2}$ M. Mulazzi, M. Hochstasser, J. Fujii, I. Vobornik, G. Rossi, and J. Henk, EPL 82, 57001 (2008).

${ }^{3}$ Photoemission and the Electronic Properties of Surfaces, edited by B. Feuerbacher, B. Fitton, and R. F. Willis (Wiley, New York, 1978); Photoemission in Solids, edited by M. Cardona and L. Ley (Springer, Berlin, 1978), Vol. 1; J. E. Inglesfield, Rep. Prog. Phys. 45, 223 (1982); R. Courths and S. Hüfner, Phys. Rep. 112, 53 (1984); Angle-Resolved Photoemission, Theory and Current Applications, edited by S. D. Kevan, Studies in Surface Science and Catalysis Vol. 74 (Elsevier, Amsterdam, 1992).

${ }^{4}$ D. Popovic, F. Reinert, S. Hüfner, V. G. Grigoryan, M. Springborg, H. Cercellier, Y. Fagot-Revurat, B. Kierren, and D. Malterre, Phys. Rev. B 72, 045419 (2005).

${ }^{5}$ R. Matzdorf, G. Meister, and A. Goldmann, Phys. Rev. B 54, 14807 (1996).

${ }^{6}$ J. Henk, M. Hoesch, J. Osterwalder, A. Ernst, and P. Bruno, J.
}

Phys.: Condens. Matter 16, 7581 (2004).

${ }^{7}$ F. Reinert and S. Hüfner, New J. Phys. 7, 97 (2005)

${ }^{8}$ M. B. Nielsen, Z. Li, S. Lizzit, A. Goldoni, and Ph. Hofmann, J. Phys.: Condens. Matter 15, 6919 (2003).

${ }^{9}$ S. D. Kevan, Phys. Rev. Lett. 50, 526 (1983).

${ }^{10}$ S. G. Louie, P. Thiry, R. Pinchaux, Y. Petroff, D. Chandesris, and J. Lecante, Phys. Rev. Lett. 44, 549 (1980).

${ }^{11}$ S. D. Kevan and R. H. Gaylord, Phys. Rev. B 36, 5809 (1987).

${ }^{12}$ A. Eyers, F. Schäfers, G. Schönhense, U. Heinzmann, H. P. Oepen, K. Hünlich, J. Kirschner, and G. Borstel, Phys. Rev. Lett. 52, 1559 (1984).

${ }^{13}$ C. M. Schneider, J. Garbe, K. Bethke, and J. Kirschner, Phys. Rev. B 39, 1031 (1989).

${ }^{14}$ J. Garbe and J. Kirschner, Phys. Rev. B 39, 1530 (1989).

${ }^{15}$ F. Baumberger, T. Greber, and J. Osterwalder, Phys. Rev. B 64, 195411 (2001)

${ }^{16}$ P. Hohenberg and W. Kohn, Phys. Rev. 136, B864 (1964); W. Kohn and L. J. Sham, ibid. 140, A1133 (1965); L. J. Sham and W. Kohn, ibid. 145, 561 (1966). 
${ }^{17}$ R. O. Jones and O. Gunnarsson, Rev. Mod. Phys. 61, 689 (1989).

${ }^{18}$ J. B. Pendry, Low Energy Electron Diffraction (Academic, London, 1974).

${ }^{19}$ J. B. Pendry, Surf. Sci. 57, 679 (1976).

${ }^{20}$ J. F. L. Hopkinson, J. B. Pendry, and D. J. Titterington, Comput. Phys. Commun. 19, 69 (1980).

${ }^{21}$ S. Hüfner, Photoelectron Spectroscopy (Springer, New York, 2003).

${ }^{22}$ A. K. Rajagopal and J. Callaway, Phys. Rev. B 7, 1912 (1973).

${ }^{23}$ M. V. Ramana and A. K. Rajagopal, Adv. Chem. Phys. 54, 231 (1983).

${ }^{24}$ F. Calogero, Variable Phase Approach to Potential Scattering (Academic, New York, 1967).

${ }^{25}$ H. Ebert and B. L. Gyorffy, J. Phys. F: Met. Phys. 18, 451 (1988).

${ }^{26}$ A. Gonis, in Green Functions for Ordered and Disordered Systems, Studies in Mathematical Physics Vol. 4 (North Holland, Amsterdam, 1992).

${ }^{27}$ X. Wang, X. G. Zhang, W. H. Butler, G. M. Stocks, and B. N. Harmon, Phys. Rev. B 46, 9352 (1992).

${ }^{28}$ S. C. Lovatt, B. L. Gyorffy, and G. Y. Guo, J. Phys.: Condens. Matter 5, 8005 (1993).

${ }^{29}$ W. Kohn and N. Rostoker, Phys. Rev. 94, 1111 (1954).
${ }^{30}$ A. R. Williams and J. van W. Morgan, J. Phys. C 7, 37 (1974).

${ }^{31}$ R. Feder, J. Phys. C 14, 2049 (1981).

${ }^{32}$ R. G. Brown and M. Ciftan, Phys. Rev. B 27, 4564 (1983).

${ }^{33}$ A. Gonis, X. G. Zhang, and D. M. Nicholson, Phys. Rev. B 40, 947 (1989)

${ }^{34}$ X. G. Zhang, A. Gonis, and J. M. MacLaren, Phys. Rev. B 40, 3694 (1989).

${ }^{35}$ W. H. Butler and R. K. Nesbet, Phys. Rev. B 42, 1518 (1990).

${ }^{36}$ R. K. Nesbet, Phys. Rev. B 41, 4948 (1990).

${ }^{37}$ W. H. Butler, A. Gonis, and X. G. Zhang, Phys. Rev. B 45, 11527 (1992).

${ }^{38}$ J. Braun, Rep. Prog. Phys. 59, 1267 (1996).

${ }^{39}$ M. Grass, J. Braun, G. Borstel, R. Schneider, H. Dürr, Th. Fauster, and V. Dose, J. Phys.: Condens. Matter 5, 599 (1993).

${ }^{40} \mathrm{H}$. Ebert, in Electronic Structure and Physical Properties of Sol$i d s$, edited by H. Dreysse, Lecture Notes in Physics Vol. 535 (Springer, Berlin, 2000), p. 191.

${ }^{41}$ G. Malmström and J. Rundgren, Comput. Phys. Commun. 19, 263 (1980).

${ }^{42}$ F. Reinert, G. Nicolay, S. Schmidt, D. Ehm, and S. Hüfner, Phys. Rev. B 63, 115415 (2001).

${ }^{43}$ B. A. McDougall, T. Balasubramanian, and E. Jensen, Phys. Rev. B 51, 13891 (1995).

${ }^{44}$ R. Eder and H. Winter, Phys. Rev. B 70, 085413 (2004). 